\title{
Effects of zolpidem on sleep parameters in patients with cirrhosis and sleep disturbances: A randomized, placebo-controlled trial
}

\author{
Manoj Kumar Sharma 1,*, Sumeet Kainth", \\ Rakhi Maiwall', Kapil Dev Jamwal', Saggere Muralikrishna Shasthry', Ankur Jindal', Ashok Choudhary', \\ Lovkesh Anand', Rajender Mal Dhamija ${ }^{4}$, Guresh Kumar ${ }^{5}$, Barjesh Chander Sharma', and Shiv Kumar Sarin ${ }^{1}$ \\ Departments of ${ }^{1}$ Hepatology and Liver Transplantation, ${ }^{2}$ Pulmonary Medicine, ${ }^{3}$ Clinical Research, ${ }^{4}$ Neurology, and ${ }^{5}$ Biostatistics, \\ Institute of Liver and Biliary Sciences, New Delhi, India
}

Background/Aims: The aim of this study was to study the efficacy and safety of zolpidem for sleep disturbances in patients with cirrhosis.

Methods: Fifty-two Child-Turcotte-Pugh (CTP) class A or B cirrhotics with Pittsburgh Sleep Quality Index $>5$ were randomized to either zolpidem $5 \mathrm{mg}$ daily $(\mathrm{n}=26)$ or placebo $(\mathrm{n}=26)$ for 4 weeks.

Results: The therapy of 4 weeks was completed by 23 patients receiving zolpidem ( 3 stopped treatment due to excessive daytime drowsiness) and 24 receiving placebo ( 2 refused to continue the study). In the zolpidem group, after 4 weeks of therapy, there was significant increase in total sleep time (TST) and sleep efficiency compared to baseline and improvement in polysomnographic parameters of sleep initiation and maintenance (i.e., decrease in sleep latency time, decrease in wake time, and decreases in number of arousals and periodic limbs movements per hour of sleep), without any significant change in sleep architecture.

Conclusions: Four weeks of $5 \mathrm{mg}$ daily zolpidem in CTP class A or B cirrhosis patients with insomnia led to significant increases in TST and sleep efficiency and improvement in polysomnographic parameters of sleep initiation and maintenance without any significant change in sleep architecture. (Clin Mol Hepatol 2019;25:199-209)

Keywords: Cirrhosis; Sleep; Zolpidem; Insomnia

\footnotetext{
Study Highlights

Sleep disturbances are common in patients with cirrhosis. Pharmacological therapies should balance hypnotic effects and adverse effects. This is the first randomized, placebo-controlled trial of zolpidem in cirrhotics with sleep disturbances. This study shows that short-term (4 week) use of zolpidem $5 \mathrm{mg}$ daily can be useful in Child-Turcotte-Pugh class A or B cirrhotics in improving total sleep time, sleep efficiency, and the parameters of sleep initiation and maintenance without any significant change in sleep architecture.
}

\footnotetext{
Abbreviations:

AHI, apnea-hypopnea index; CFF, critical flicker frequency; CTP, Child-TurcottePugh; ESS, Epworth Sleepiness Scale; HE, hepatic encephalopathy; ILBS, Institute of Liver and Biliary Sciences; MHE, minimal hepatic encephalopathy; OSA, obstructive sleep apnea; PLMS, periodic limbs movements of sleep; PSG, polysomnography; PSQI, Pittsburgh Sleep Quality Index; REM, rapid eye movement; TRT, total recording time; TST, total sleep time

\section{Corresponding author : Manoj Kumar Sharma}

Department of Hepatology and Liver Transplantation, Institute of Liver and Biliary Sciences, Sector D1 Vasant Kunj, New Delhi 110070, India Tel: +91-11-46300000, Fax: +91-11-46300063

E-mail: manojkumardm@gmail.com https://orcid.org/0000-0003-3448-8334 


\section{INTRODUCTION}

Patients with cirrhosis often complain of sleep difficulties even without overt encephalopathy. ${ }^{1,2}$ Management of sleep difficulties in these patients is problematic. Pharmacological therapies must achieve a balance between hypnotic and adverse effects. Zolpidem is a high-affinity positive modulator of $\omega 1 \mathrm{GABA}_{A}$ receptors. ${ }^{3}$ The mean half-life of zolpidem in cirrhotic patients of $9.9 \mathrm{hr}$ (range: 4.1 to $25.8 \mathrm{hr}$ ) was greater than that observed in normal subjects of $2.2 \mathrm{hr}$ (range: 1.6 to $2.4 \mathrm{hr}$ ). ${ }^{4}$

The recommended dose for immediate release formulation of zolpidem is $10 \mathrm{mg}$ in adult males, $5 \mathrm{mg}$ in adult women, and 5 mg in elderly and patients with hepatic impairment. ${ }^{4}$ In 2011, a sublingual, lower-dose tablet was approved, with doses that differed for men and women $\left(3.5 \mathrm{mg}\right.$ for men and $1.75 \mathrm{mg}$ for women. ${ }^{5}$

There are no published randomized controlled studies on efficacy and safety of zolpidem in patients with cirrhosis. The aim of this study was to study the efficacy and safety of zolpidem for sleep disturbances in patients with cirrhosis.

\section{MATERIALS AND METHODS}

\section{Trial design}

This was a single center, double-blind, randomized placebo-controlled trial (ClinicalTrials.gov identifier number: NCT02484963).

\section{Participants}

The study was conducted in the Department of Hepatology and Liver Transplantation, Institute of Liver and Biliary Sciences (ILBS), New Delhi from 30/6/2015 to 20/12/2016. The study was approved by the ILBS Institutional Review Board (Number: F. 25/5/75/ILBS/AC/2014/392). Informed consent was taken from the participants and the work was done in accordance with the declaration of Helsinki.

Patients who fulfilled the following inclusion criteria were eligible to participate in the study: age 18 to 70 years, Child-TurcottePugh (CTP) class A and B cirrhosis (CTP score up to 9) of any etiology, and history of persistent sleep disturbance with Pittsburgh Sleep Quality Index (PSQI) $>5$.

Exclusion criteria were the following: active alcohol intake or intake within 1 month of enrollment, active substance abuse or intake within 1 month of enrollment, known psychiatric and neuro- logical disorders, patients using antidepressant, anticonvulsants, other hypnotics, pregnancy or lactation, hepatocellular carcinoma, prior history of overt hepatic encephalopathy or currently in overt encephalopathy, patients at high risk of obstructive sleep apnea (OSA), CTP class C cirrhosis, acute decompensated state of cirrhosis like gastrointestinal bleed/increased jaundice, active infection, post transjugular intrahepatic portosystemic shunt patients, acute febrile illness and no consent.

\section{Interventions}

After fulfilling all inclusion and exclusion criteria patients were randomized to either zolpidem ( $5 \mathrm{mg}$ per oral 30 minutes before sleeping at night daily) or placebo (peroral at bed time daily) for 4 weeks. The same dose of $5 \mathrm{mg}$ of zolpidem was used for both males and females.

All patients were advised regarding good sleep hygiene.

Both the group of patients underwent the following investigations at baseline: hemogram, renal and liver function tests, serum electrolytes, etiological workup for cirrhosis as needed, abdominal ultrasound with Doppler splenoportal axis and hepatic veins, blood sugar fasting, chest X-ray, arterial ammonia level, critical flicker frequency (CFF), PSQI, Epworth Sleepiness Scale (ESS), and polysomnography (PSG).

Patients were followed up weekly up to fourth week of therapy. During each follow up visit following were assessed: compliance, any adverse drug effect, renal and liver function tests, arterial ammonia levels. At the fourth week, in addition, PSQI score assessment, ESS, CFF and PSG were repeated.

The patients and their relatives were instructed to contact the investigators immediately in the event of any alteration in the patient's mental state, excessive sleepiness or drowsiness. Patients were strictly advised not to drive during the study period.

\section{Outcomes}

The primary outcome measure was improvement in total sleep time (TST) as measured by PSG at end of 4 weeks. The secondary outcome measures studied at end of 4 weeks were: change in other polysomnographic parameters, improvement in PSQI score, precipitation of hepatic encephalopathy, or development of any other side effects. 


\section{Randomization and allocation concealment}

Random allocation sequence was done by computer-generated random number codes with an equal number of the alternative treatment. Patients were randomized to either of the two groups in 1:1 ratio. Sealed opaque thick papered envelopes were used to conceal the sequence until interventions were assigned.

\section{Blinding}

Treatment and placebo pills were of similar size, shape and color. The participants, care providers, and those assessing outcomes were blinded. The code was not broken until the end of the study.

\section{Medication preparation}

The Institute Pharmacy procured and prepared the study medication in size \#1 opaque capsules. Zolpidem capsules contained $5 \mathrm{mg}$ of zolpidem and placebo capsules (identical in appearance to zolpidem capsules) contained starch.

\section{Compliance}

Pills were supplied to the patients for 1 week (7 pills) at one time, and patients and attendants were instructed to taken only one pill per day. Compliance was checked every week by interview and pill count technique. The same dose was continued throughout the trial period (no dose titration was done), with the plan to discontinue the patient in case major adverse events occur (excessive sedation, neuropsychiatric side effects or encephalopathy).

\section{Study assessments}

\section{PSQI and ESS}

The PSQI is a self-administered questionnaire, with a global PSQI score greater than 5 indicating a "poor" sleeper. ${ }^{6}$ ESS is used to assess day-time sleepiness, with values higher than 10 reflects above normal daytime sleepiness. ${ }^{7}$

PSQI and ESS has been validated in the Indian population ${ }^{8-10}$ and cirrhotic populations. ${ }^{11,12}$ Overall PSQI global score and ESS score correlation coefficient for test-retest reliability has been found to be $\operatorname{good}(>0.80)^{13,14}$

\section{Measurement of CFF threshold}

CFF analysis was done by HEP Atonorm analyzer (Accelab
$\mathrm{GmbH}$, Kusterdingen, Germany). ${ }^{15}$ Patients were diagnosed as minimal hepatic encephalopathy (MHE) if mean of 8 CFF readings was $<39 \mathrm{~Hz}^{15}$

\section{PSG}

All patients were subjected to complete overnight PSG in the sleep unit of the Department of Pulmonology, Institute of Liver and Biliary Sciences, New Delhi, India, using the sleep laboratory digital system EMBLA $57000^{\circledR}$ (Embla Systems Inc., Broomfield, CO, USA). After an initial adaptation night, PSG was performed on the second night.

The subjects were instructed to go to sleep at their usual bedtime. All PSG sessions were monitored by trained technicians and visually scored according to standardized criteria. ${ }^{16}$

The following PSG sleep parameters were recorded; (1) total recording time (TRT), (2) TST, (3) wake time, (4) sleep efficiency (the TST/TRT ratio, expressed as percentage), (5) sleep latency: the length of time to sleep onset, (6) latency to rapid eye movement (REM) sleep, (7) percentage of time patients spent in sleep stages 1, 2, $3+4$ or REM sleep stage, (8) apnea-hypopnea index (AHI): mean number of apneas or hypopneas in a 1-h period, (9) periodic limbs movements of sleep per hour (PLMS/h), (10) arousals/h, (11) mean Sp02: the mean oxy-hemoglobin saturation, and (12) nadir Sp02: minimal oxy-hemoglobin saturation recorded during PSG.

An abnormal $\mathrm{AHI}$ ( $\mathrm{AHI}$ of more than five events per hour of sleep) accompanied by excessive daytime sleepiness were diagnosed as OSA. ${ }^{17}$

PSG was conducted by the neurology team. These parameters were analyzed and scored by blinded specialists (Kumar S, Agarwal HK, and Dhamija RM) and the scores were separately tabulated by all three (with intra-class correlation coefficient of more than 0.9 for all variables) and finally the mean of the three was taken as final value of each variable in the PSG report.

\section{Statistical methods}

Data was processed using the software packages SPSS version 20.0 (IBM Corp., Armonk, NY, USA). For comparison of categorical variables, chi-square and Fisher's exact tests were used. For comparision of continuous variables, $t$-test for normally distributed continous variables and Mann-Whitney $U$ test for continous variables not normally distributed were used. Wilcoxon rank sum test for paired continuous data, and McNemar test for paired categorical variables were used. 


\section{Sample size}

Zolpidem has been shown to be effective from the very first day (in non-cirrhotic subjects), in trials of zolpidem use in transient insomnia including trials using the first night effect as a model of transient insomnia, ${ }^{18}$ therefore use of PSQI questionnaire (which covers the 4 weeks prior to assessment) as an assessment tool of this 4-week drug trial is justified. The primary outcome measure was improvement in TST as measured by PSG at end of 4 weeks. As there was no prior data on Indian cirrhotic patients, we initially did PSG on 10 patients with cirrhosis with CTP A and B (without the exclusion criteria for the study as above) and mean TST of $248.3 \pm 64.4 \mathrm{~min}$. Assuming baseline TST to be $240 \pm 60 \mathrm{~min}$ in each group and that zolpidem and placebo will increase TST by $120 \mathrm{~min}$ (to $360 \pm 60 \mathrm{~min}$ ) and $60 \mathrm{~min}$ (to $300 \pm 60 \mathrm{~min}$ ) respec- tively; with alpha $5 \%$ and power $90 \%$ we needed to enroll 22 cases in each arm of the study. Assuming defaulter rate to be $10 \%$ it was decided to enroll total 50 cases ( 25 cases in each group). No interim analysis was planned.

\section{RESULTS}

\section{Participant flow and numbers analyzed}

945 patients with cirrhosis, without overt encephalopathy were screened for the presence of sleep disturbances, by administering PSQI and ESS (Fig. 1).

Finally, 52 patients who fulfilled the inclusion and exclusion criteria were randomized to the zolpidem arm $(n=26)$ and placebo

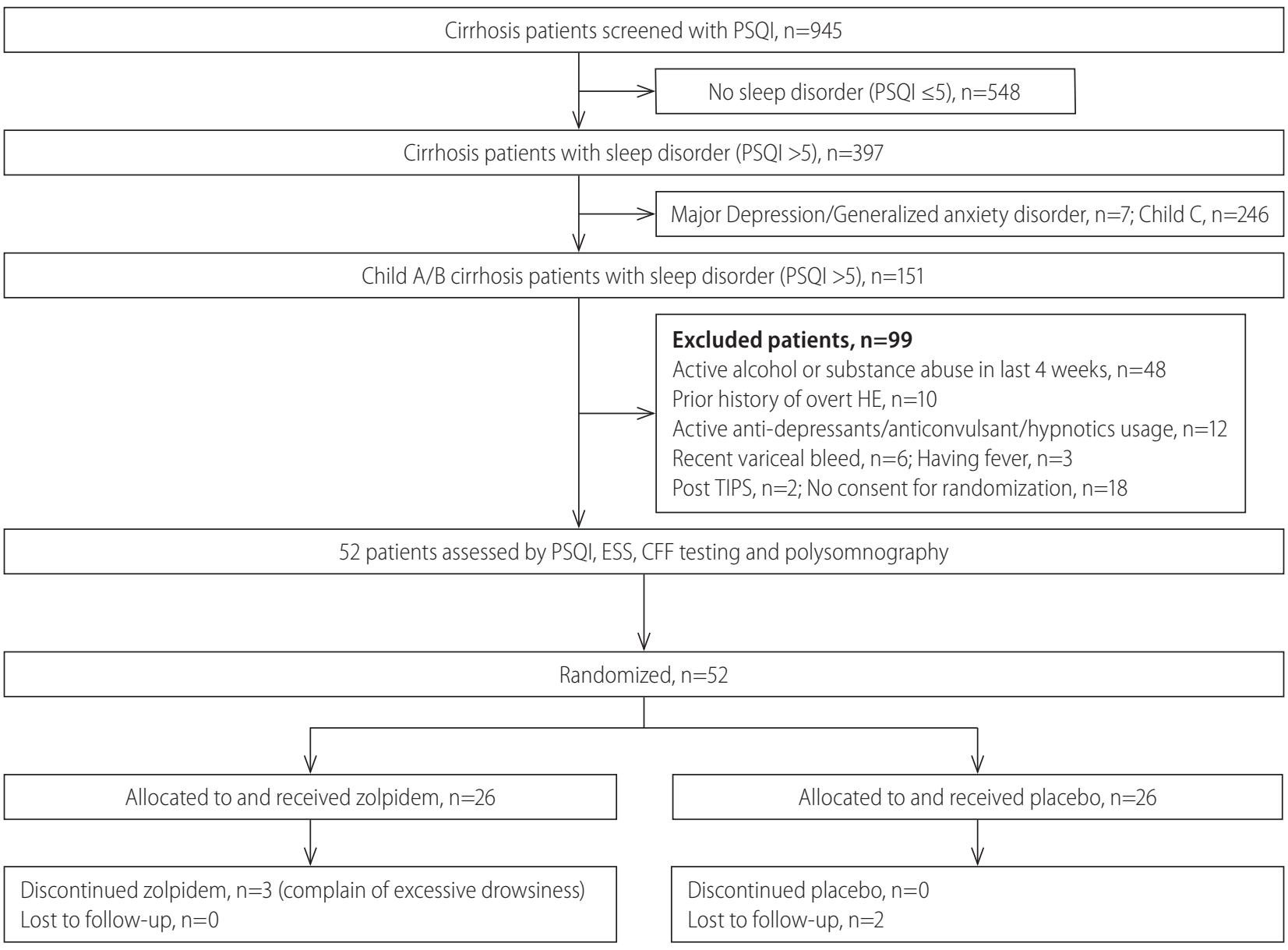

Figure 1. Participant flow in the study. Nine-hundred forty five patients with cirrhosis, without overt encephalopathy were screened for the presence of sleep disturbances. Finally 52 patients who fulfilled the inclusion and exclusion criteria were randomized to the zolpidem arm ( $n=26)$ and placebo arms $(n=26)$. None of the patients were lost to follow-up in zolpidem arm, where as 2 patients were lost to follow-up in the placebo arm. PSQI, Pittsburgh Sleep Quality Index; HE, hepatic encephalopathy; TIPS, transjugular intrahepatic portosystemic shunt; ESS, Epworth Sleepiness Scale; CFF, critical flicker frequency. 
arms $(n=26)$. Standard medical care was continued in both the arms, which included beta-blockers and diuretics. As patients with prior history of overt hepatic encephalopathy were excluded, none of the patients were on lactulose, rifaximin or L-ornithine-Laspartate. None of the patients were treated with antibiotics during the study period.

\section{Baseline data}

Tables 1 and 2 show the baseline demographic and clinical characteristics for each group.

The therapy of 4 weeks was completed by 23 patients in zolpidem group (3 patients stopped treatment before completion of 4 weeks due to development of excessive daytime drowsiness) and 24 patients in placebo group (2 patients refused to continue in the study).

\section{Changes in PSG findings after 4 weeks of therapy}

\section{Overall assessments of zolpidem efficacy}

In the zolpidem group, after 4 weeks of therapy, as compared to baseline, there was significant increase in TST (from median [range] of 245.5 [120.3-370.5] min to 370.3 [180.0-435.5] min, $P \leq 0.001$ in zolpidem vs. $251.8(123.3-365.5) \mathrm{min}$ to 200.4 (70.5-438.5) $\mathrm{min}, P=0.440$ in placebo]; and increase in sleep efficiency (from median [range] of 68.4 [19.8-87.9]\% to 78.9 [60.0-92.2]\%, $P \leq 0.001$ in zolpidem vs. $69.8[19.9-90.7] \%$ to $68.9[10.8-227.8] \%, P=1.000$ in placebo).

\section{Sleep initiation and maintenance}

In the zolpidem group, after 4 weeks of therapy, as compared to baseline, there was significant decrease in sleep latency time, decrease in number of arousals per hour of sleep; decrease in wake time; and decrease in number of periodic limbs movements of sleep per hour (Table 3).

Table 1. Baseline demographic and clinical characteristics of patients receiving zolpidem and placebo

\begin{tabular}{|c|c|c|c|}
\hline Parameters & Zolpidem ( $n=26)$ & Placebo $(n=26)$ & $P$-value \\
\hline Age (years) & $53.7 \pm 10.2$ & $55.3 \pm 8.0$ & 0.520 \\
\hline Male sex (n, \%) & $22(84.6)$ & $22(84.6)$ & 0.857 \\
\hline \multicolumn{4}{|l|}{ Etiology of cirrhosis (n, \%) } \\
\hline NASH/Alcohol/HBV/HCV & $13(50) / 10(38.5) / 2(7.7) / 1(3.8)$ & $12(46.2) / 13(50) / 0 / 1(3.8)$ & 0.546 \\
\hline $\operatorname{BMI}\left(\mathrm{kg} / \mathrm{m}^{2}\right)$ & $26.1 \pm 5.2$ & $26.8 \pm 5.6$ & 0.348 \\
\hline $\mathrm{Hb}(\mathrm{g} / \mathrm{dL})$ & $10.6 \pm 1.7$ & $10.4 \pm 1.7$ & 0.646 \\
\hline TLC (per micro L) & $6,731.3 \pm 2,333.7$ & $6,488.4 \pm 3,991.4$ & 0.790 \\
\hline Platelet $\left(10^{9} / \mathrm{L}\right)$ (median, range) & $100.5(68.7-140.0)$ & $97.5(81.5-110.3)$ & 0.833 \\
\hline Creatinine (mg/dL) & $0.76 \pm 0.23$ & $0.83 \pm 0.27$ & 0.348 \\
\hline Bilirubin (mg/dL) & $2.25 \pm 1.67$ & $1.73 \pm 1.65$ & 0.274 \\
\hline Serum albumin (g/L) & $3.4 \pm 0.6$ & $3.2 \pm 0.5$ & 0.326 \\
\hline INR & $1.64 \pm 0.66$ & $1.81 \pm 0.96$ & 0.486 \\
\hline \multicolumn{4}{|l|}{ CTP class (n, \%) } \\
\hline$A / B$ & $12(46.2) / 14(53.8)$ & $12(46.2) / 14(53.8)$ & 0.981 \\
\hline CTP score & $6.96 \pm 1.34$ & $6.85 \pm 1.15$ & 0.741 \\
\hline MELD & $14.5 \pm 4.9$ & $12.9 \pm 2.6$ & 0.145 \\
\hline Art. NH3 $(\mu \mathrm{g} / \mathrm{dL})$ & $94.9 \pm 19.1$ & $105.1 \pm 31.1$ & 0.164 \\
\hline \multicolumn{4}{|l|}{ Concomitant medications (n, \%) } \\
\hline Diuretics & $11(42.3)$ & $13(50)$ & 0.781 \\
\hline Beta blockers & $14(53.8)$ & $14(53.8)$ & 1.000 \\
\hline
\end{tabular}

Values are presented as mean \pm standard deviation unless otherwise indicated.

NASH, nonalcoholic steatohepatitis; HBV, hepatitis B virus; HCV, hepatitis C virus; BMI, body mass index; Hb, hemoglobin; TLC, total leukocyte count; INR, International Normalized Ratio; CTP, Child-Turcotte-Pugh; MELD, model for end-stage liver disease; Art. NH3, arterial ammonia. 
Table 2. Critical flicker frequency, sleep and anxiety/depression questionnaires, and polysomnographic findings at baseline in patients receiving zolpidem or placebo

\begin{tabular}{|c|c|c|c|}
\hline Parameters & Zolpidem ( $n=26)$ & Placebo $(n=26)$ & $P$-value \\
\hline \multicolumn{4}{|c|}{ Measures of minimal hepatic encephalopathy } \\
\hline $\mathrm{CFF}(\mathrm{Hz})$ & $39.2(33.6-42.6)$ & $39.7(32.8-43.0)$ & 0.647 \\
\hline CFF $<39 \mathrm{~Hz}(\mathrm{n}, \%)$ & $13(50)$ & $10(38.5)$ & 0.577 \\
\hline \multicolumn{4}{|l|}{ Sleep quality } \\
\hline PSQI score & $14.0(8.0-18.0)$ & $14.0(10.0-16.0)$ & 0.701 \\
\hline \multicolumn{4}{|l|}{ Day-time sleepiness } \\
\hline ESS score & $6.5(0.0-18.0)$ & $6.5(0.0-16.0)$ & 0.242 \\
\hline ESS score >10 (n, \%) & $3(11.5)$ & $4(15.3)$ & 0.684 \\
\hline \multicolumn{4}{|l|}{ Polysomnography } \\
\hline \multicolumn{4}{|l|}{ Overall sleep quality } \\
\hline TRT (min) & $335.8(173.5-961.0)$ & $328.4(159.3-495.4)$ & 0.080 \\
\hline TST (min) & $245.5(120.3-370.5)$ & $251.8(123.3-365.5)$ & 0.833 \\
\hline SE (\%) & $68.4(19.8-87.9)$ & $69.8(19.9-90.7)$ & 0.456 \\
\hline \multicolumn{4}{|l|}{ Sleep initiation and maintenance } \\
\hline Sleep Lat (min) & $65.3(35.2-180.3)$ & $65.4(30.2-120.5)$ & 0.464 \\
\hline Arousals (/h) & $3.0(2.0-3.0)$ & $3.0(0.0-3.0)$ & 0.152 \\
\hline Wake* (min) & $108.9(34.5-676.5)$ & $98.5(35.6-456.8)$ & 0.767 \\
\hline $\operatorname{PLMS}(/ \mathrm{h})$ & $29.6(0.0-179.0)$ & $27.3(0.0-151.0)$ & 0.365 \\
\hline \multicolumn{4}{|l|}{ Sleep architecture } \\
\hline REM Lat (min) & $110.3(56.0-189.5)$ & $103.3(53.4-154.0)$ & 0.589 \\
\hline Sleep time in stage 1 (\%) & $15.9(1.1-43.6)$ & $15.8(1.4-60.0)$ & 0.667 \\
\hline Sleep time in stage $2(\%)$ & $46.7(13.0-71.6)$ & $37.7(3.7-67.3)$ & 0.094 \\
\hline Sleep time in stage $3+4(\%)$ & $15.2(2.4-50.2)$ & $26.7(0.7-53.7)$ & 0.126 \\
\hline Sleep time in REM (\%) & $10.0(1.6-49.2)$ & $14.2(0.8-64.2)$ & 0.146 \\
\hline \multicolumn{4}{|l|}{ Indices of apnea-hypopnea } \\
\hline AHI (events/h) & $0.7(0.0-3.7)$ & $0.9(0.0-5.0)$ & 0.134 \\
\hline $\mathrm{AHI}>5 / \mathrm{h}(\mathrm{n}, \%)$ & $0(0)$ & $0(0)$ & 1.000 \\
\hline OSA (n, \%) & $0(0)$ & $0(0)$ & 1.000 \\
\hline Mean SpO2 (\%) & $95.5(88.0-99.0)$ & $95.2(90.0-98.0)$ & 0.352 \\
\hline Nadir SpO2 (\%) & $90.0(83.0-95.0)$ & $90.5(81.5-94.0)$ & 0.534 \\
\hline
\end{tabular}

Values are presented as median (range) unless otherwise indicated.

CFF, critical flicker frequency; PSQI, Pittsburgh sleep quality index; ESS, Epworth Sleepiness Scale; TRT, total recording time; TST, total sleep time; SE, sleep efficiency; Sleep Lat, latency to sleep onset; PLMS, periodic limbs movements of sleep; REM Lat, rapid eye movement sleep latency; AHI, apnea-hypopnea index; OSA, obstructive sleep apnea; Sp02, oxy-hemoglobin saturation; Nadir Sp02, minimal oxy-hemoglobin saturation.

*Minutes awake after sleep onset.

\section{Sleep architecture and indices of apnea-hypopnea}

After 4 weeks of therapy, as compared to baseline, there was no significant change in sleep architecture or indices of apnea-hypopnea (Table 3).

\section{Changes in PSQI and ESS after 4 weeks of therapy}

After 4 weeks of therapy, as compared to baseline, there was significant improvement in PSQI score in zolpidem group but not in placebo group (Table 3). 
Table 3. Comparison of sleep parameters after 4 weeks of therapy in zolpidem and placebo groups

\begin{tabular}{|c|c|c|c|c|c|}
\hline Parameters & Zolpidem ( $n=23)$ & Placebo $(n=24)$ & P1 value $^{\dagger}$ & P2 value ${ }^{\ddagger}$ & P3 value ${ }^{\S}$ \\
\hline \multicolumn{6}{|l|}{ Polysomnography } \\
\hline \multicolumn{6}{|l|}{ Overall sleep quality } \\
\hline TRT (min) & $450.5(235.0-630.5)$ & $288.9(93.3-483.0)$ & $<0.001$ & 0.078 & 0.511 \\
\hline TST (min) & $370.3(180.0-435.5)$ & $200.4(70.5-438.5)$ & $<0.001$ & $<0.001$ & 0.440 \\
\hline SE (\%) & $78.9(60.0-92.2)$ & $68.9(10.8-227.8)$ & 0.006 & $<0.001$ & 1.000 \\
\hline \multicolumn{6}{|l|}{ Sleep initiation and maintenance } \\
\hline Sleep Lat (min) & $30.5(15.0-125.3)$ & $71.4(15.4-110.4)$ & 0.001 & $<0.001$ & 0.943 \\
\hline Arousals (/h) & $1.0(0.0-3.0)$ & $3.0(1.0-3.0)$ & $<0.001$ & $<0.001$ & 0.942 \\
\hline Wake* (min) & $82.6(30.3-231.4)$ & $74.7(20.4-130.0)$ & 0.125 & 0.052 & 0.909 \\
\hline $\operatorname{PLMS}(/ \mathrm{h})$ & $6.8(0.0-105.5)$ & $26.5(0.0-140.5)$ & 0.001 & 0.028 & 0.131 \\
\hline \multicolumn{6}{|l|}{ Sleep architecture } \\
\hline REM Lat (min) & $90.4(64.5-134.2)$ & $95.4(64.3-144.3)$ & 0.873 & 0.230 & 0.063 \\
\hline Sleep time in stage 1 (\%) & $12.3(1.4-36.3)$ & $15.9(2.2-67.0)$ & 0.489 & 0.301 & 0.219 \\
\hline Sleep time in stage $2(\%)$ & $46.6(13.0-71.6)$ & $37.9(6.1-68.1)$ & 0.167 & 0.548 & 0.841 \\
\hline Sleep time in stage $3+4(\%)$ & $17.0(0.0-58.5)$ & $25.8(0.0-61.7)$ & 0.338 & 0.784 & 0.819 \\
\hline Sleep time in REM (\%) & $15.2(2.4-50.2)$ & $10.8(1.2-56.3)$ & 0.307 & 0.131 & 0.204 \\
\hline \multicolumn{6}{|l|}{ Indices of apnea-hypopnea } \\
\hline AHI (events/h) & $0.8(0.0-3.8)$ & $0.9(0.3-6.6)$ & 0.334 & 0.519 & 0.875 \\
\hline Mean SpO2 (\%) & $96.0(91.0-99.0)$ & $95.0(91.0-99.0)$ & 0.122 & 0.981 & 0.138 \\
\hline Nadir SpO2 (\%) & $90.0(83.0-95.0)$ & $87.0(75.0-95.0)$ & 0.248 & 0.711 & 0.441 \\
\hline \multicolumn{6}{|l|}{ Minimal hepatic encephalopathy } \\
\hline $\mathrm{CFF}(\mathrm{Hz})$ & $38.4(33.4-40.6)$ & $38.8(31.4-42.6)$ & 0.647 & 0.031 & 0.394 \\
\hline CFF $<39 \mathrm{~Hz}(\mathrm{n}, \%)$ & $15(65.2)$ & $14(58.3)$ & 0.746 & 0.375 & 0.219 \\
\hline Art. NH3 ( $\mu \mathrm{g} / \mathrm{dL})$ (mean \pm standard deviation) & $95.6 \pm 16.7$ & $103.1 \pm 32.6$ & 0.509 & 0.939 & 0.274 \\
\hline \multicolumn{6}{|l|}{ Sleep quality score } \\
\hline PSQI score & $11.0(7.0-18)$ & $14.0(10.0-16.0)$ & 0.009 & 0.004 & 0.682 \\
\hline \% change in PQSI score & $-21.4(-50$ to +20$)$ & $0.0(-40$ to +30$)$ & 0.024 & NA & NA \\
\hline$>20 \%$ improvement in PSQI score (n, \%) & $13(56.5)$ & $3(12.5)$ & 0.002 & NA & NA \\
\hline \multicolumn{6}{|l|}{ Day-time sleepiness } \\
\hline ESS score & $6.5(0.0-18.0)$ & $6.5(0.0-16.0)$ & 0.067 & 0.098 & 0.095 \\
\hline
\end{tabular}

Values are presented as median (range) unless otherwise indicated.

TRT, total recording time; TST, total sleep time; SE, sleep efficiency; Sleep Lat, latency to sleep onset; PLMS, periodic limbs movements of sleep; REM Lat, rapid eye movement sleep latency; AHI, apnea-hypopnea index; Sp02, oxy-hemoglobin saturation; Nadir SpO2, minimal oxy-hemoglobin saturation; CFF, critical flicker frequency; Art. NH3, arterial ammonia; PSQI, Pittsburgh sleep quality index; ESS, Epworth Sleepiness Scale; NA, not applicable.

${ }^{*}$ Minutes awake after sleep onset.

${ }^{\dagger} P$-value zolipdem vs. placebo.

${ }^{\ddagger} P$-value baseline vs. 4 weeks for zolpidem group.

${ }^{\S} P$-value baseline vs. 4 weeks for placebo group.

\section{Changes in CFF threshold and arterial ammonia after 4 weeks of therapy}

In the zolpidem group, after 4 weeks of therapy, as compared to baseline, there was significant decline in CFF threshold (from median [range] of 39.2 [33.6-42.6] Hz to 38.4 [33.4-40.6] Hz, $P=0.031$ in zolpidem vs. $39.7[32.8-43.0] \mathrm{Hz}$ to $38.8[31.4-42.6]$ $\mathrm{Hz}, P=0.394$ in placebo), however, there was no significant 
change in number of patients with CFF $<39 \mathrm{~Hz}$ in either of the groups (Table 3). There was no significant change in arterial ammonia levels in either group after 4 weeks of therapy (Table 3).

\section{Side effects of therapy}

Three of 26 (11.5\%) patients in zolpidem group had excessive day time drowsiness (that improved within 24 hours of stoppage of therapy), as compared to none in the placebo group (Table 4). The clinical profile of the 3 patients who developed excessive day time drowsiness is shown in Table 5.

Other side effects are shown in Table 4. None of the patients developed overt hepatic encephalopathy, hallucinations or other neuropsychiatric manifestation or complex behaviors during study period. Constipation was seen more frequently in zolpidem-treated patients (23.1\%) as compared to placebo-treated patients $(3.8 \%, P=0.054)$, which was managed by increased fiber intake in food in all and in none of them lactulose or lactitol was used.

\section{DISCUSSION}

This is the first double-blind, placebo-controlled randomized PSG trial assessing safety and efficacy of zolpidem in patients with cirrhosis and insomnia. In the zolpidem group, after 4 weeks of therapy, as compared to baseline, there was significant increase in TST (from median [range] of 245.5 [120.3-370.5] min to 370.3 [180.0-435.5] min, $P \leq 0.001$ in zolpidem vs. 251.8 [123.3$365.5] \mathrm{min}$ to 200.4 [70.5-438.5] min, $P=0.440$ in placebo); and increase in sleep efficiency (from median [range] of 68.4 [19.8$87.9] \%$ to $78.9[60.0-92.2] \%, P \leq 0.001$ in zolpidem vs. 69.8
[19.9-90.7]\% to $68.9[10.8-227.8] \%, P=1.000$ in placebo). There was also improvement in polysomnographic parameters of sleep initiation and maintenance (i.e., decrease in sleep latency time decrease in number of arousals per hour of sleep, decrease in wake time, and decrease in number of periodic limbs movements of sleep per hour). Overall, these findings suggest that zolpidem leads to improvement in overall sleep quality (improvememt in TST and sleep efficiency), decrease in time to initiation of sleep and is better able to maintain the sleep through the night. However the sleep architecture did not change. Also, after 4 weeks of therapy, as compared to baseline, there was significant improvement in subjective assessment of sleep quality by the patients as assessed by the PSQI score in zolpidem group. These findings are similar to other studies of zolpidem in non-cirrhotic patients. ${ }^{19,20}$

Till date, only a few studies have been done using different drugs for sleep disturbances in patients with cirrhosis. In a study including 35 patients with cirrhosis and MHE and long-standing sleep difficulties, patients were randomized to histamine $H_{1}$ blocker, hydroxyzine $25 \mathrm{mg}$ at bedtime $(n=17)$ or placebo $(n=18)$ for a 10-day period. Objectively, $65 \%$ of hydroxyzine-treated patients vs. $25 \%$ of patients under placebo had $\mathrm{a} \geq 30 \%$ increase in sleep efficiency as measured by wrist actigraphy $(P=0.041)$. One patient developed an acute episode of encephalopathy which was reversible upon cessation of hydroxyzine. ${ }^{21}$

In one study which assessed the effect of rifaximin for 28 days on sleep architecture parameters in 15 cirrhotic patients with recurrent hepatic encephalopathy (HE), it was found that on 24-h PSG, rifaximin improved HE scores, improved objective sleep architecture parameters, with no changes in the subjective quality of sleep and sleepiness. ${ }^{22}$

In our study, $50 \%$ of those in the zolpidem group and $38.5 \%$ in

Table 4. Side effects of therapy in zolpidem and placebo groups

\begin{tabular}{lccc}
\hline Side effects & Zolpidem $(\mathbf{n = 2 6 )}$ & Placebo $(\mathbf{n = 2 6 )}$ & $\boldsymbol{P}$-value \\
\hline Excessive day time drowsiness & $3(11.5)$ & $0(0)$ & 0.086 \\
\hline Tireness & $9(34.6)$ & $6(23.1)$ & 0.459 \\
\hline Headache & $2(7.7)$ & $0(0)$ & 0.166 \\
\hline Bodyaches & $5(19.2)$ & $4(15.4)$ & 0.814 \\
Nausea/Vomitings & $1(3.8)$ & $0(0)$ & 0.332 \\
Diarrhoea & $1(3.8)$ & $0(0)$ & 0.332 \\
Constipation & $6(23.1)$ & $1(3.8)$ & 0.054 \\
Dry mouth & $3(11.5)$ & $0(0)$ & 0.086 \\
Hepatic encephalopathy & $0(0)$ & $0(0)$ & 1.000 \\
\hline
\end{tabular}

Values are presented as $n(\%)$ unless otherwise indicated. 
the placebo group had minimal HE (based on CFF <39). Although there was significant decline in CFF threshold in zolpidem group at week 4 as compared to baseline; there was no significant change in number of patients with CFF $<39 \mathrm{~Hz}$ in either zolpidem or placebo groups; and none of the patients treated with zolpidem developed overt HE. One report reviewing development of HE during zolpidem use ${ }^{23}$ reported that since the approval of zolpidem in 1992 through September 2015, the Food and Drug Administration identified six spontaneous cases of HE associated with zolpidem in patients with hepatic insufficiency. Our study is not powered to detect development of significant side effects like overt HE development.

One intriguing finding in this study was that zolpidem reduced the number of periodic limb movements of sleep per hour. In fact there are some reports of development of periodic limb movement during zolpidem treatment especially in elderly females. ${ }^{24}$ On the other hand, zolpidem has been found to be effective in a variety of movement disorders. ${ }^{25}$

Constipation was seen more frequently in zolpidem-treated pa- tients (23.1\%) as compared to placebo-treated patients $(3.8 \%$, $P=0.054)$. This finding is intriguing as previous studies in non-cirrhotics have found constipation in around $2 \%$ of zolpidem-treated patients. $^{4}$

The three patients who stopped zolpidem treatment due to severe daytime sleepiness were either female or old or (in case of patient 1) showed in addition definite signs of liver insufficiency. Perhaps female and older patients with cirrhosis may be started with a lower $2.5 \mathrm{mg}$ dose instead of $5 \mathrm{mg} /$ day dose.

However, the present study has a few limitations. The zolpidem groups were not significantly different from each other in study attrition, but the reasons for discontinuations were different. The placebo group had more voluntary discontinuations $(n=2)$ than the zolpidem group $(n=0)$. These discontinuations may reflect the participant's ability to discern that the study medication had little beneficial effect on their sleep and were more likely to stop taking study medication and discontinue the study. In the zolpidem group, all the discontinuations were due to excessive daytime sleepiness $(n=3)$.

Table 5. The clinical profiles of the 3 patients who developed excessive day time drowsiness on zolpidem therapy

\begin{tabular}{|c|c|c|c|}
\hline & Patient 1 & Patient 2 & Patient 3 \\
\hline Age (years) & 70 & 50 & 61 \\
\hline Sex & Male & Female & Female \\
\hline Etiology of cirrhosis & NASH & $\mathrm{NASH}$ & $\mathrm{NASH}$ \\
\hline $\mathrm{BMI}\left(\mathrm{kg} / \mathrm{m}^{2}\right)$ & 24.7 & 28.9 & 35.9 \\
\hline $\mathrm{Hb}(\mathrm{g} / \mathrm{dL})$ & 8.6 & 10.1 & 13.0 \\
\hline Creatinine (mg/dL) (mean+standard deviation) & 0.9 & 0.6 & 0.34 \\
\hline Serum sodium (meq/L) & 129 & 132 & 139 \\
\hline Serum potassium (meq/L) & 4.7 & 4.2 & 4.1 \\
\hline Bilirubin (mg/dL) & 5.4 & 2.1 & 1.2 \\
\hline AST (IU/L) & 90 & 24 & 24 \\
\hline ALT (IU/L) & 74 & 20 & 23 \\
\hline Serum albumin (g/L) & 3.5 & 3.5 & 3.3 \\
\hline INR & 1.92 & 1.23 & 1.16 \\
\hline CTP score & 8 & 5 & 7 \\
\hline MELD & 20 & 12 & 9 \\
\hline $\mathrm{TSH}(\mathrm{U} / \mathrm{mL})$ & 4.5 & 4.5 & 4.8 \\
\hline Art. NH3 ( $\mu \mathrm{g} / \mathrm{dL})$ & 111 & 78 & 71 \\
\hline Concomitant medications & Beta blockers & No beta blockers or diuretics & Beta blockers, Diuretics \\
\hline $\mathrm{CFF}(\mathrm{Hz})$ & 42 & 40.1 & 33.6 \\
\hline
\end{tabular}

NASH, nonalcoholic steatohepatitis; BMI, body mass index; $\mathrm{Hb}$, hemoglobin; AST, aspartate aminotransferase; ALT, alanine aminotransferase; INR, International Normalized Ratio; CTP, Child-Turcotte-Pugh; MELD, model for end-stage liver disease; TSH, thyroid stimulating hormone; Art. NH3, arterial ammonia; CFF, critical flicker frequency. 
Only CTP class A and B cirrhosis (CTP score upto 9) were included. The safety in CTP class C cirrhosis remains unknown.

Zopidem is a drug with many concerns related to adverse effects. Especially in patients with hepatic impairment, gamma-aminobutyric acid agonists, such as zopidem, are well known as important precipitating factors for hepatic encephalopathy. The study has a relatively small number of patients to assess this safety. Also, a 4-week trial is insufficient to determine the safety of zolpidem in cirrhosis patients. A longer period and larger trial would be needed to uncover significant safety concerns. Another limitation of this study was that we did not use wrist actigraphy, which is less precise that PSG but has the the advantage of monitoring subjects while they perform their customary social activities, avoiding the constraints of the sleep laboratory. Also, this study did not study the improvement in daytime functioning and quality of life after therapy.

Four weeks of $5 \mathrm{mg}$ daily zolpidem in CTP class A and B cirrhosis with insomnia leads to a significant increase in TST and sleep efficiency; improvement in polysomnographic parameters of sleep initiation and maintenance (i.e., decrease in sleep latency time decrease in number of arousals per hour of sleep, decrease in wake time, and decrease in number of periodic limb movements of sleep per hour), without any significant change in sleep architecture.

\section{Authors' contribution}

Manoj Kumar Sharma, Sumeet Kainth, and Shiv Kumar Sarin developed the protocol. Manoj Kumar Sharma, Sumeet Kainth, and Ankit Bhardwaj enrolled participants in the study. Sachin Kumar, Hemant Kumar Agarwal, and Rajender Mal Dhamija interpretated the polysomnographic findings. Kapil Dev Jamwal, Rakhi Maiwall, Saggere Muralikrishna Shasthry, Ankur Jindal, Ashok Choudhary, Lovkesh Anand, and Barjesh Chander Sharma reviewed and provided inputs to the protocol and manuscript. Guresh Kumar helped in statistical analysis. All of the authors have read and approved the final manuscript.

\section{Conflicts of Interest}

The authors declare they have no conflict of interest.

\section{REFERENCES}

1. Hamdan AJ, Al Enezi A, Anwar AE, Abdullah AH, Baharoon S, Aljumah $A$, et al. Prevalence of insomnia and sleep patterns among liver cirrhosis patients. J Circadian Rhythms 2014;12:2.

2. Montagnese S, Middleton B, Skene DJ, Morgan MY. Night-time sleep disturbance does not correlate with neuropsychiatric impairment in patients with cirrhosis. Liver Int 2009;29:1372-1382.

3. Che Has AT, Absalom N, van Nieuwenhuijzen PS, Clarkson AN, Ahring PK, Chebib M. Zolpidem is a potent stoichiometry-selective modulator of a1 $\beta 3$ GABAA receptors: evidence of a novel benzodiazepine site in the a1-a1 interface. Sci Rep 2016;6:28674.

4. AMBIEN Prescribing information, Sanofi-Aventis U.S. LLC. NDA 19908 S027 FDA approved labeling 4.23.08. Food and Drug Administration (FDA) web site, <https://www.accessdata.fda.gov/ drugsatfda_docs/label/2008/019908s027lbl.pdf>. Accessed 10 Jan 2018.

5. Norman JL, Fixen DR, Saseen JJ, Saba LM, Linnebur SA. Zolpidem prescribing practices before and after Food and Drug Administration required product labeling changes. SAGE Open Med 2017;5:2050312117707687.

6. Buysse DJ, Reynolds CF 3rd, Monk TH, Berman SR, Kupfer DJ. The Pittsburgh Sleep Quality Index: a new instrument for psychiatric practice and research. Psychiatry Res 1989;28:193-213.

7. Johns MW. A new method for measuring daytime sleepiness: the Epworth sleepiness scale. Sleep 1991;14:540-545.

8. De S. Subjective assessment of quality of sleep in chronic obstructive pulmonary disease patient and its relationship with associated depression. Lung India 2012;29:332-335.

9. Singh J, Sharma BC, Puri V, Sachdeva S, Srivastava S. Sleep disturbances in patients of liver cirrhosis with minimal hepatic encephalopathy before and after lactulose therapy. Metab Brain Dis 2017:32:595-605.

10. Manzar MD, Moiz JA, Zannat W, Spence DW, Pandi-Perumal SR, Ba Hammam AS, et al. Validity of the Pittsburgh Sleep Quality Index in Indian university students. Oman Med J 2015;30:193-202.

11. Stewart CA, Auger RR, Enders FT, Felmlee-Devine D, Smith GE. The effects of poor sleep quality on cognitive function of patients with cirrhosis. J Clin Sleep Med 2014;10:21-26.

12. Mostacci B, Ferlisi M, Baldi Antognini A, Sama C, Morelli C, Mondini $S$, et al. Sleep disturbance and daytime sleepiness in patients with cirrhosis: a case control study. Neurol Sci 2008;29:237-240.

13. Backhaus J, Junghanns K, Broocks A, Riemann D, Hohagen F. Testretest reliability and validity of the Pittsburgh Sleep Quality Index in primary insomnia. J Psychosom Res 2002;53:737-740.

14. Johns MW. Reliability and factor analysis of the Epworth Sleepiness Scale. Sleep 1992;15:376-381.

15. Sharma P, Sharma BC, Puri V, Sarin SK. Critical flicker frequency: diagnostic tool for minimal hepatic encephalopathy. J Hepatol 2007;47:67-73.

16. Hori T, Sugita Y, Koga E, Shirakawa S, Inoue K, Uchida S, et al. Proposed supplements and amendments to 'A Manual of Standardized 
Terminology, Techniques and Scoring System for Sleep Stages of Human Subjects', the Rechtschaffen \& Kales (1968) standard. Psychiatry Clin Neurosci 2001;55:305-310.

17. Medical Advisory Secretariat. Polysomnography in patients with obstructive sleep apnea: an evidence-based analysis. Ont Health Technol Assess Ser 2006;6:1-38.

18. Roth T, Roehrs T, Vogel G. Zolpidem in the treatment of transient insomnia: a double-blind, randomized comparison with placebo. Sleep 1995;18:246-251.

19. Monti JM. Effect of zolpidem on sleep in insomniac patients. Eur J Clin Pharmacol 1989;36:461-466.

20. Scharf MB, Roth T, Vogel GW, Walsh JK. A multicenter, placebocontrolled study evaluating zolpidem in the treatment of chronic insomnia. J Clin Psychiatry 1994;55:192-199.

21. Spahr L, Coeytaux A, Giostra E, Hadengue A, Annoni JM. Histamine $\mathrm{H} 1$ blocker hydroxyzine improves sleep in patients with cirrhosis and minimal hepatic encephalopathy: a randomized controlled pilot trial.
Am J Gastroenterol 2007;102:744-753.

22. Bruyneel M, Sersté $T$, Libert $W$, van den Broecke $S$, Ameye L, Dachy $B$, et al. Improvement of sleep architecture parameters in cirrhotic patients with recurrent hepatic encephalopathy with the use of rifaximin. Eur I Gastroenterol Hepatol 2017;29:302-308.

23. Nevo ON, Brinker AD, Diak IL, Kortepeter CM. Response to: Improvement of sleep architecture parameters in cirrhotic patients with recurrent hepatic encephalopathy with the use of rifaximin: hepatic encephalopathy in association with zolpidem. Eur J Gastroenterol Hepatol 2017;29:1102-1103.

24. eHealthMe. Who have Periodic limb movement disorder with Zolpidem - from FDA reports. eHealthMe web site, <https://www. ehealthme.com/ds/zolpidem/periodic-limb-movement-disorder>. Accessed 12 Jan 2018.

25. Bomalaski MN, Claflin ES, Townsend W, Peterson MD. Zolpidem for the treatment of neurologic disorders: a systematic review. JAMA Neurol 2017;74:1130-1139. 